\title{
Nano-meter Scale Observation of Local Network Structure in Aluminosilicate Glass via Vibrational EELS
}

Kunyen Liao ${ }^{1}$, Atsunobu Masuno ${ }^{2}$, Hiroyuki Inoue ${ }^{1}$ and Teruyasu Mizoguchi ${ }^{3}$

${ }^{1}$ The University of Tokyo, Tokyo, Tokyo, Japan, ${ }^{2}$ Hirosaki University, Hirosaki, Aomori, Japan, ${ }^{3}$ The University of Tokyo, Meguro-ku, Tokyo, Japan

Understanding atomic arrangement and its orderings in network glass is crucial to develop new glassy materials for future applications in optics and electronics. In recent years, great interest has been attracted to aluminosilicate glass, well-known as the crack-resistant glass at the displays. The conventional description of its structure is based on the random network theory, where both $\mathrm{Si}$ and $\mathrm{Al}$ cations form tetrahedral networks through the bridging oxygen (BO) [1]. However, several works in recent years have shown the presence of unusual amounts of highly coordinated 5-fold and 6-fold Al in the structure, accompanied with formation of triply coordinated oxygens, tricluster oxygens (TO) [2]. Such coordination changes at $\mathrm{Al}$ and $\mathrm{O}$ atoms may be the origin of various superior properties like high hardness and crackresistance [3]. Understanding the mechanism behind is thus crucial toward the design of stronger glasses.

While nuclear magnetic resonance (NMR) and X-ray total scattering are widely used for structural analysis of silicate glasses, they provide little spatial resolution to study the local coordination within nanostructures such as phase separation, which has been reported in several aluminosilicate glasses. How 5-fold, 6-fold Al and TO are distributed and interplayed with each other in a phase-separated microstructure is still a mystery. A high-resolution chemical and structural analysis is thus essential to access a comprehensive understanding on the structure.

In this work, we investigated the local coordination of $\mathrm{Al}$ and $\mathrm{O}$ in a phase-separated aluminosilicate glass using electron energy loss spectroscopy (EELS) at scanning transmission electron microscopy (STEM). Binary aluminosilicate glass with composition $50 \mathrm{Al}_{2} \mathrm{O}_{3}-50 \mathrm{SiO}_{2}$ was studied with an aberrationcorrected STEM (JEM-ARM200F, JEOL Ltd.). Figure 1(a) and (b) show the high angle annular dark field (HAADF) image and bright field (BF) image of the specimen. The intensity of HAADF and BF are modulated by formation of droplet-like domains with the size around 20 40nm. Elemental mapping was performed using the Al-L2,3 edge (around 77eV) and Si-L2,3 edge (around 110eV), as shown in Fig.1(c) and (d). The fine structure of the Al-L2,3 edge was further simulated with density functional theory (DFT) calculation. The comparison between the experimental and the theoretical spectra allowed the identification of differently coordinated Al sites in spectra. The result was further used for mapping $\mathrm{Al}$ coordination in real space.

On the other hand, information on $\mathrm{O}$ coordination is hard to obtain from the $\mathrm{O} \mathrm{K}$ absorption edge. Here, we utilized newly developed monochromatic EELS to record atomic vibration in the network glass. Stretching mode of $\mathrm{SiO}_{4}$ group was investigated with the support of DFT phonon calculation and molecular dynamics (MD) simulation. According to the energy difference of local stretching mode in two separated phases, we can distinguish the differently coordinated $\mathrm{O}$ at real space, as shown in Fig.2. For the first time, the presence of tricluster oxygens is experimentally confirmed since it was proposed three decades ago. The details of analysis will be given in my presentation. 

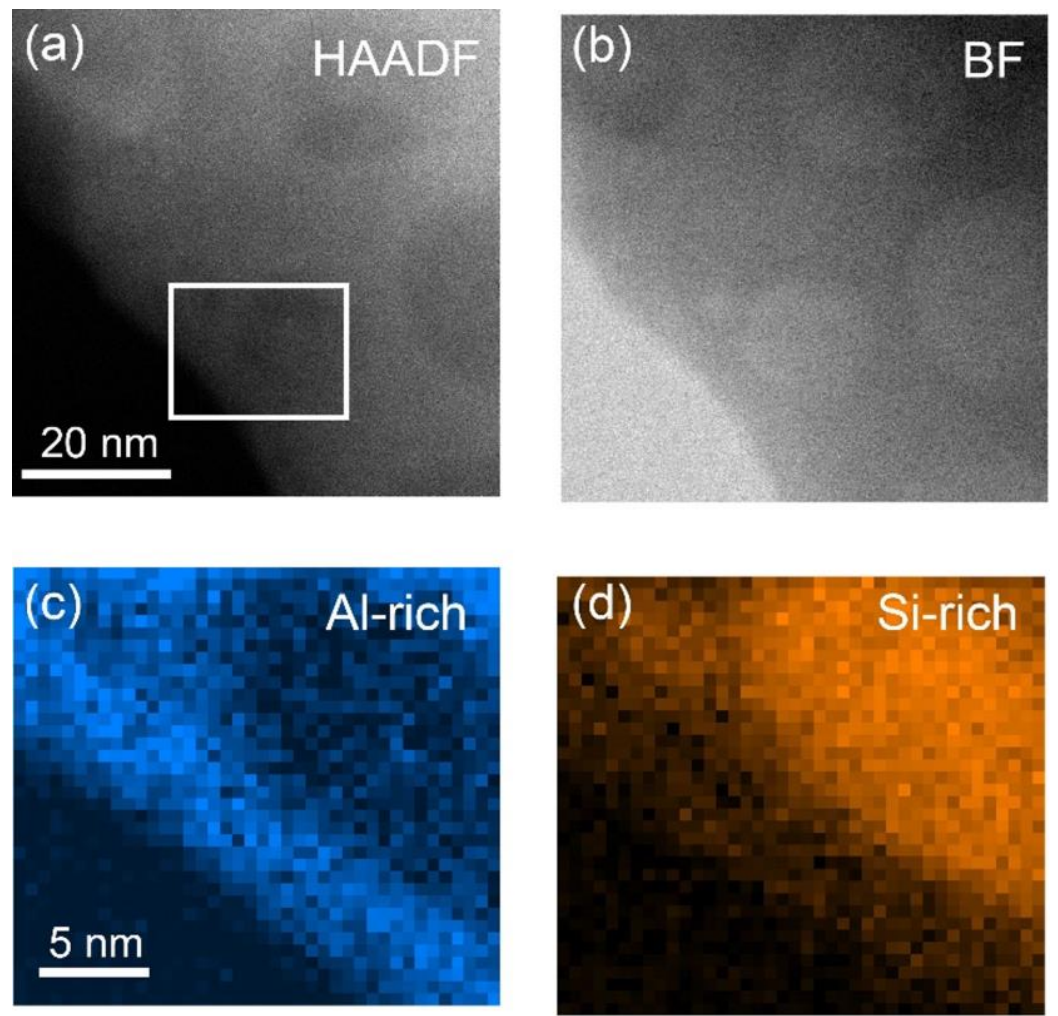

Figure 1. STEM observation of aluminosilicate glasses. (a) HAADF image (b) BF image of phase separated regions. (c) and (d) show EELS chemical mapping of $\mathrm{Al}$ and $\mathrm{Si}$ L2,3 edge respectively.

(a)

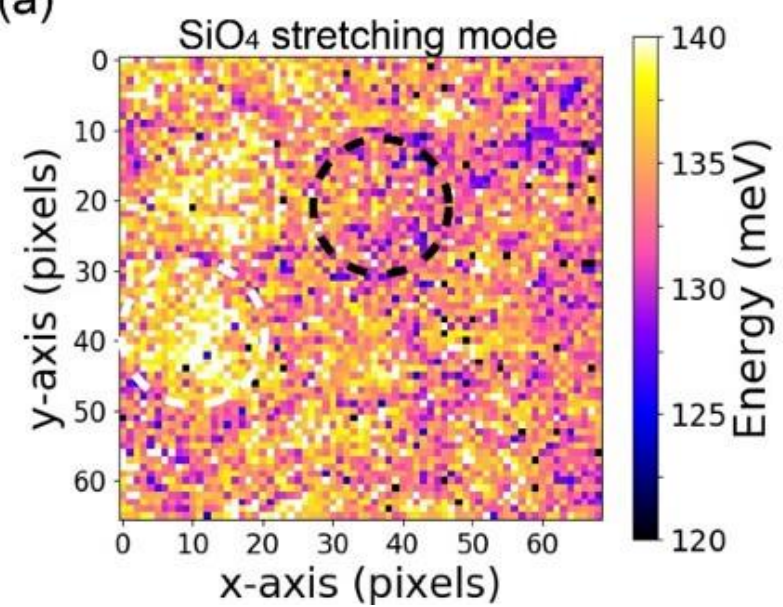

(b)

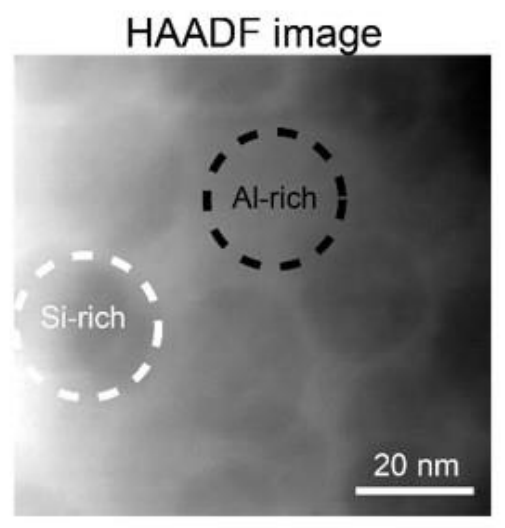

Figure 2. Vibrational mode mapping of $\mathrm{SiO} 4$ asymmetric stretching modes. (a) Mapping of $\mathrm{SiO} 4$ peak position obtained from profile fitting. Size of each pixel is $1.4 \mathrm{~nm}$ times $1.4 \mathrm{~nm}$. The difference of color reflects the variation of local $\mathrm{O}$ coordination. (b) HAADF image of selected regions.

References

[1] Merzbacher, C. I et al., Journal of Non-Crystalline Solids, 130, 18-34 (1991).

[2] Weber. R et al., J. Phys. Chem. B 112, 16726-16733 (2008).

[3] Gustavo A. R et al., Sci. Rep. 6, 23620 (2016). 